\title{
In-situ Lorentz TEM Observations in Ferromagnetic Shape Memory Alloys
}

\author{
S.P. Venkateswaran, N.T. Nuhfer, and M. De Graef \\ Department of Materials Science \& Engineering, Carnegie Mellon University, Pittsburgh, PA 15213
}

Ferromagnetic shape memory alloys (FSMAs), such as $\mathrm{Ni}_{2} \mathrm{MnGa}$ and $\mathrm{Co}_{2} \mathrm{NiGa}$, exhibit a thermoelastic martensitic transformation similar to conventional shape memory alloys [1]. While the mechanical aspects of this transformation have been studied extensively, the microstructural aspects of the magnetic domain structure and its evolution during the martensitic transformation have not been documented. In this study, we have explored the dynamics and reversibility of the magnetic domain structure of $\mathrm{Ni}_{2} \mathrm{MnGa}$ by means of in-situ Lorentz Transmission Electron Microscopy (LTEM).

Stoichiometric $\mathrm{Ni}_{2} \mathrm{MnGa}$ has a Curie temperature of $376 \mathrm{~K}$ and a martensitic temperature $\mathrm{M}_{s}=202 \mathrm{~K}$ [1]. Its high temperature austenitic phase has a Heusler $\left(\mathrm{L} 22_{1}\right)$ structure and a low magnetocrystalline anisotropy, while the low temperature martensitic phase is tetragonal with a high magnetocrystalline anisotropy [2]. In this study, an off-stoichiometric composition of $\mathrm{Ni}_{2} \mathrm{Mn}_{1+x} \mathrm{Ga}_{1-x}\left(x=0.08, \mathrm{M}_{s}=\right.$ $260 \mathrm{~K}$ ) was chosen because of its near room temperature transformation. In-situ LTEM studies were performed using a Tecnai F20 field emission microscope equipped with a Lorentz piece and a Gatan double tilt liquid nitrogen cooling holder $\left(1^{\circ} \mathrm{C} / \mathrm{min}\right)$. All thin foils were prepared by electropolishing. Fresnel (out-of-focus) images were recorded using a CCD camera through a Gatan Imaging Filter operated in zero loss mode with a $14 \mathrm{eV}$ energy selecting slit.

Fig. 1 shows typical Fresnel images (underfocus mode) of the magnetic domain structure in both the austenite and martensite phases. The white and the dark contrast (indicated by the arrows) represents the magnetic domain walls. While the austenite phase (Fig. 1(a)) displays curved domain walls, in accordance with its low magnetocrystalline anisotropy, the martensite phase (Fig. 1(b)) shows much straighter domain walls, in agreement with its high magnetocrystalline anisotropy. Note the presence of domain walls coinciding with the twin boundaries as well as jagged domain walls running across the twin domains. The austenitic phase in Fig. 1(b) has not yet completely transformed to martensite.

Fig. 2 (a) through (c) show Fresnel (underfocus) images of the magnetic domain structure during cooling. As the sample is cooled below $\mathrm{M}_{s}$, the martensite plates begin to form and the domain walls rearrange themselves, as dictated by the magnetocrystalline anisotropy of the martensite phase. As the sample is heated back to room temperature (Fig. 2 (d) through (f)), the plates retrace their path confirming the thermoelastic nature of the martensitic transformation. In addition to the structural reversibility, it can be seen that the magnetic domain configuration also reverts back to the same shape (compare the arrowed locations in Figs. 2(a) and (f)), signifying that the domain structure is completely reversible. The origin of this reversibility is currently under investigation. It is also interesting to note that upon approaching the martensitic transformation temperature from above, both structural tweed image contrast and magnetoelastic tweed contrast are present.

\section{References}

1. P.J. Webster, K.R.A. Ziebeck, S.L. Town, \& M.S. Peak, Phil. Mag. B, 49 (1984) 295.

2. K. Ullako, J. Huang, C. Kantner, R. O’Handley, \& V.V. Kokorin, Appl. Phys. Lett., 69 (1996) 1966.

3. This work was supported by the U.S. National Science Foundation (DMR-0404836). 

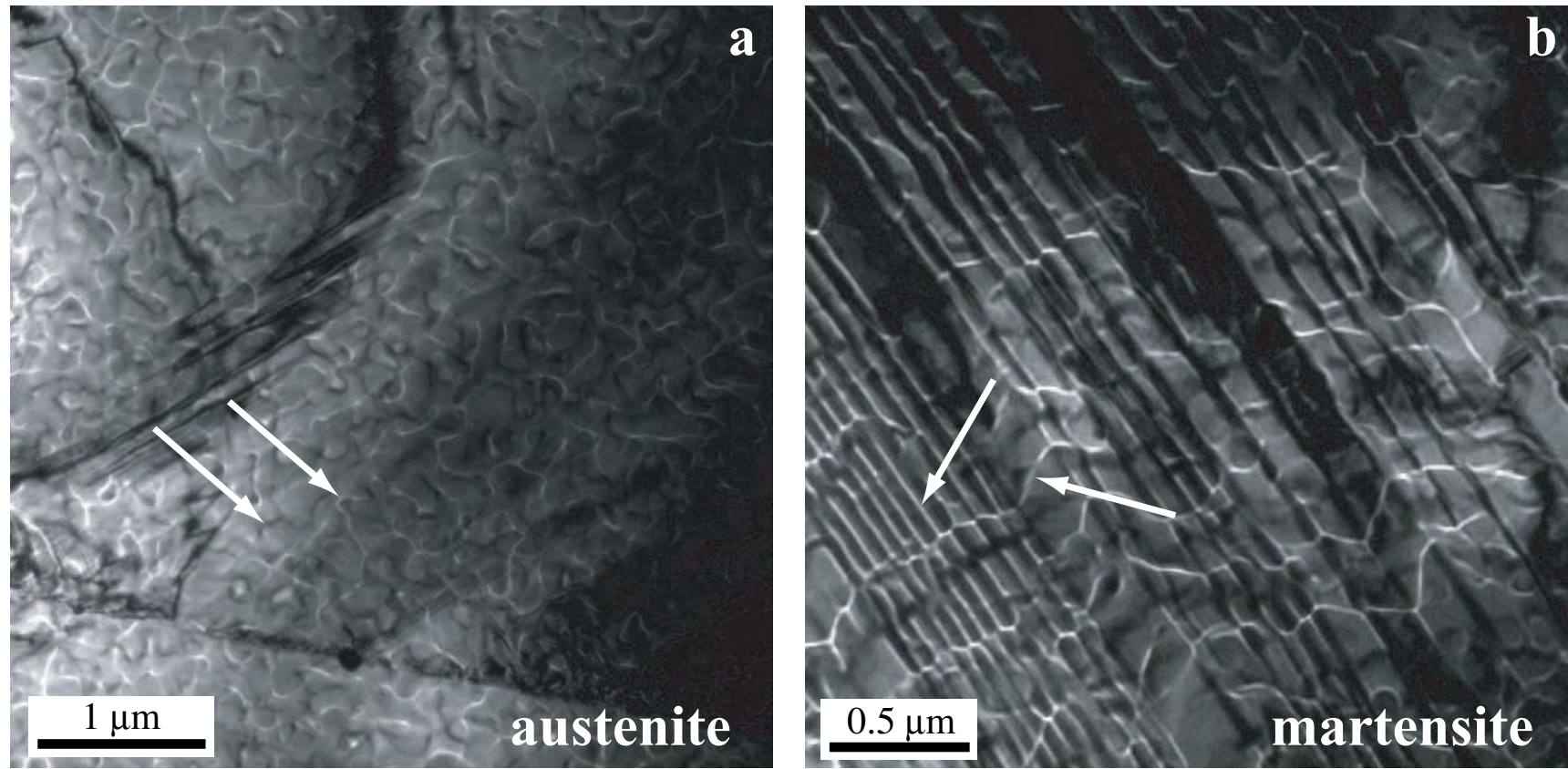

Figure 1. Fresnel (underfocus) images of austenite and partially transformed martensite. Magnetic domain walls are arrowed.
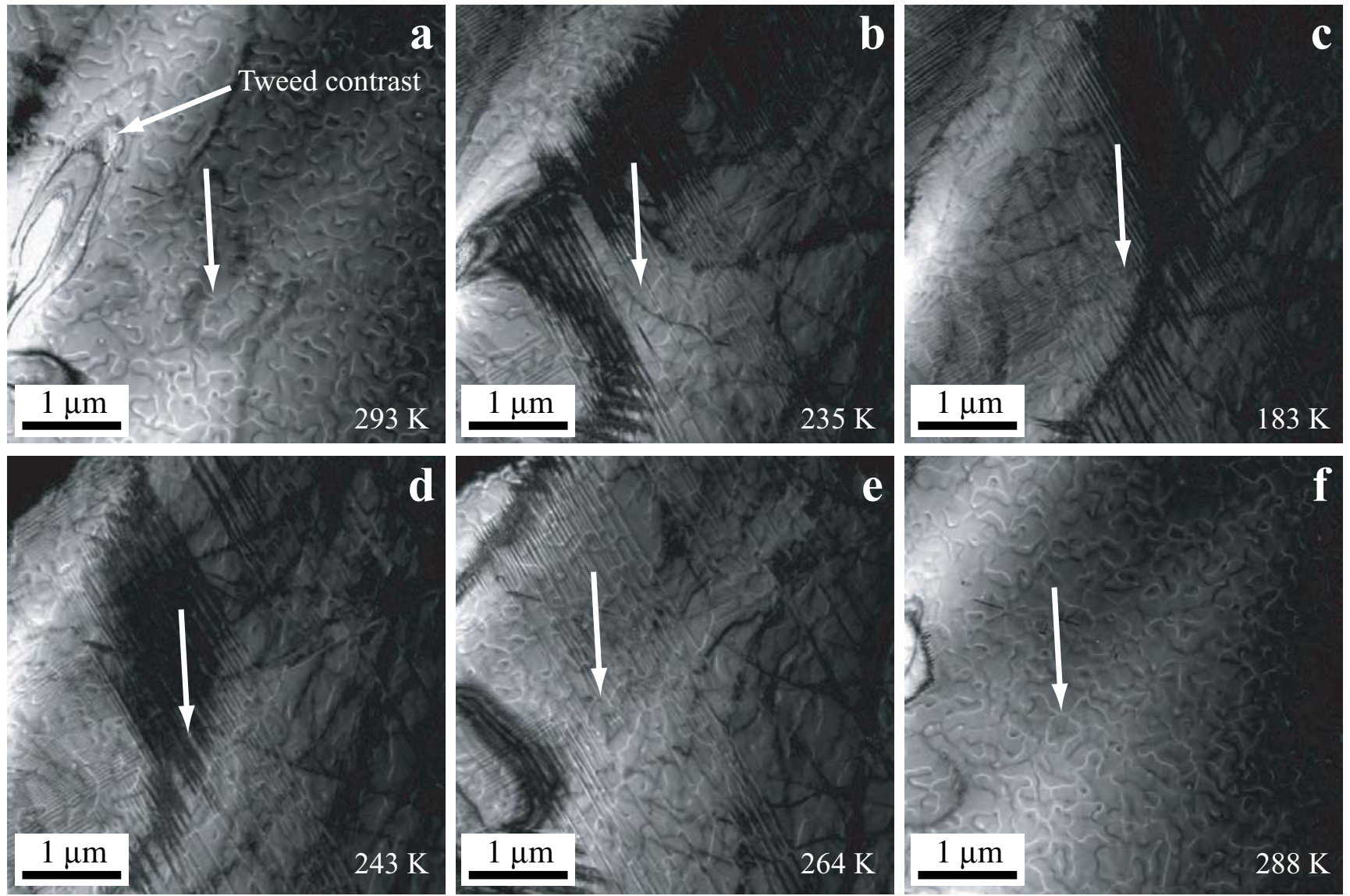

Figure 2. a)-c) Magnetic domain structure at different temperatures during cooling; d)-f) Domain structure during heating. Domain structure reversibility is shown by arrows. 